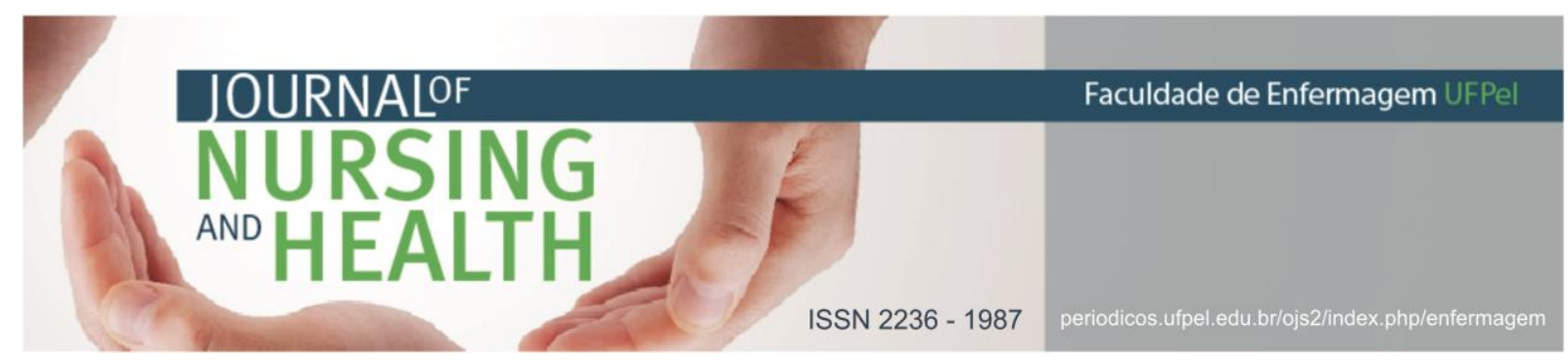

ARTIGO ORIGINAL

\title{
Perfil das idosas atendidas por queda em um serviço de emergência
}

\author{
Profile of aged women attended by accidental falls in emergency health units
}

\author{
Perfil de ancianas atendidas por caída en un servicio de emergencia
}

Daiane Lopes Leal BORBA ${ }^{1}$, Celmira LANGE ${ }^{2}$, Patrícia Mirapalheta Pereira LLANO ${ }^{3}$, Samanta Bastos
MAAGH ${ }^{4}$, Aline da Costa VIEGAS ${ }^{5}$, Bianca Pozza dos SANTOS

\begin{abstract}
RESUMO
Objetivo: identificar o perfil das idosas atendidas por queda em um serviço de emergência no sul do Brasil. Métodos: estudo quantitativo e descritivo. A coleta de dados ocorreu de março a abril de 2010. Os sujeitos foram 153 idosas. Resultados: as idosas com idade mais avançada, faixa etária entre 7080 anos e brancas foram as que mais sofreram quedas; metade era viúva e possuía renda de até um salário mínimo; mais de dois terços referiram saber ler. A maioria apresentava hipertensão arterial, ${ }^{1}$ reumatismo, problemas de memória, nervosismo e visão. Majoritariamente tinham dificuldade para caminhar e utilizavam polifármacos. Ainda, mais de $60 \%$ sofreram pelo menos uma queda no ano anterior. Conclusão: os profissionais da saúde devem estar capacitados e preparados para atender à idosa que sofreu acidente por queda, de maneira individualizada e singular, promovendo ações eficazes, orientando novos hábitos, melhorando a condição física, diminuindo assim, os riscos de queda.
\end{abstract}

Descritores: Idoso; Mulheres; Acidentes por quedas; Enfermagem.

\section{ABSTRACT}

Objective: to identify the profile of aged women attended by accidental falls in an emergency health unit in the south of Brazil. Methods: quantitative and descriptive study. Data collection occurred since March to April of 2010. Subjects were 153 aged women. Results: the aged women had between 70-80 years old, and were white; half of them were widow and had income up to a minimum wage; more than two-thirds referred that they knew how to read. The most of them presented arterial hypertension, rheumatism, memory problems, jitters and vision; had difficulty to walk and took

\footnotetext{
${ }^{1}$ Enfermeira. Enfermeira Supervisora do Núcleo Materno Infantil do Hospital São Francisco de Paula. Pelotas, Rio Grande do Sul(RS), Brasil. Email: daianelleal@yahoo.com.br

2 Enfermeira. Doutora em Ciências. Professora Associada da Faculdade de Enfermagem e do Programa de Pós Graduação em Enfermagem da Universidade Federal de Pelotas. Pelotas, RS, Brasil. Email: celmira_lange@terra.com.br

${ }^{3}$ Enfermeira. Doutora em Ciências. Colaboradora da Empresa Servando e Fhurmman LTDA Pelotas, Pelotas, RS, Brasil. Email: patihepp@yahoo.com.br

${ }^{4}$ Enfermeira. Mestre em Ciências. Docente da Faculdade de Enfermagem da Universidade Católica de Pelotas e Enfermeira Assistencial do Programa de Internação Domiciliar Interdisciplinar do Hospital Escola da Universidade Federal de Pelotas/EBSERH. Pelotas, RS, Brasil. Email: samantamaagh@yahoo.com.br

${ }^{5}$ Enfermeira. Mestre em Ciências. Doutoranda pelo Programa de Pós Graduação em Enfermagem da Universidade Federal de Pelotas. Enfermeira Assistencial do Programa Melhor em Casa do Hospital Escola da Universidade Federal de Pelotas/EBSERH. Pelotas, RS, Brasil. Email: alinecviegas@hotmail.com

${ }^{6}$ Enfermeira. Mestre em Ciências. Doutoranda pelo Programa de Pós Graduação em Enfermagem da Universidade Federal de Pelotas. Enfermeira Assistencial da Prefeitura Municipal de Pelotas. Pelotas, RS, Brasil. Email: bi.santos@bol.com.br
} 


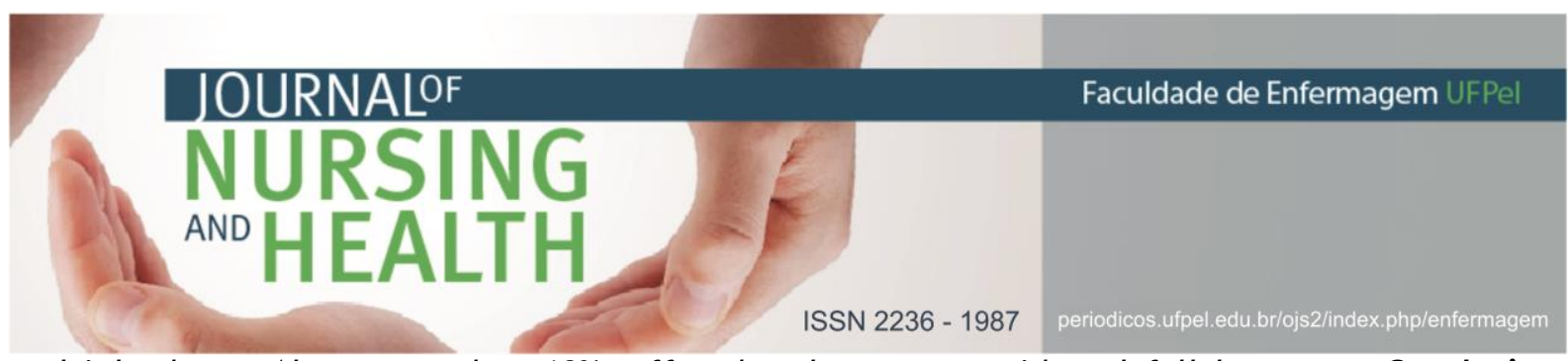

multiple drugs. Also, more than $60 \%$ suffered at least one accidental fall last year. Conclusion: health professionals must to be capable and prepared of attend to woman who suffers accidental falls, in an individualized and singular way, promoting effective actions, guiding new habits, improving physical conditions, decreasing, therefore, accidental falls risks.

Descriptors: Aged; Women; Accidental falls; Nursing.

\section{RESUMEN}

Objetivo: identificar el perfil de ancianas atendidas por caída en un servicio de emergencia en el sur de Brasil. Métodos: estudio cualitativo y descriptivo. La recolecta de datos ocurrió de marzo a abril de 2010. Los sujetos fueron 153 ancianas. Resultados: las ancianas presentaran entre 70-80 años y eran blancas; mitad era viuda y poseía renda de hasta un salario mínimo; más de dos tersos refirieran saber leer. La mayoría presentaba hipertensión arterial, reumatismo, problemas de memoria, nervios y visión; tenían dificultad para caminar y utilizaban múltiplos remedios. Aún, más de $60 \%$ sufrieran por lo menos una caída en el año pasado. Conclusión: los profesionales de salud deben estar capacitados y preparados para atender la anciana que sufrió accidente por caída, de manera individualizada y singular, promoviendo acciones eficaces, orientando nuevos hábitos, mejorando la condición física, decreciendo así, los riesgos de caída.

Descriptores: Anciano; Mujeres; Accidentes por caídas; Enfermería.

\section{INTRODUÇÃO}

No Brasil, o envelhecimento da população é confirmado por meio dos dados do censo demográfico, constatando que os idosos com idade igual ou superior a 60 anos correspondem a $11,8 \%$ da população total de 190.755.799. No Rio Grande do Sul (RS), conforme os dados do Instituto Brasileiro de Geografia e Estatística (IBGE) de 2010, 13,8\% da população sul-rio-grandense são considerados idosos, constituindo o Estado brasileiro que possui o maior número de idosos, ou seja, 10.693.929 milhões de habitantes possuem 60 anos ou mais. Já o Município de Pelotas, tem uma população de 328.275 mil habitantes, dos quais $15,3 \%$ possuem 60 anos ou mais. ${ }^{1}$

Com o avanço da ciência e o crescimento cada vez maior das tecnologias dirigidas à saúde das pessoas, nos últimos anos se observa um aumento da população idosa. Em virtude desse crescimento, há uma elevação no número de quedas nesta população. Esse fator pode ser decorrente do estilo de vida, tornandothes mais ativos, mas que os levam à exposição para o risco de acidentes. ${ }^{2}$

Os fatores de risco que predispõem a queda estão relacionados às causas intrínsecas, que se dão através da diminuição da visão e audição, do sedentarismo, dos distúrbios músculos esqueléticos, da alteração na postura, da alteração de equilíbrio e locomoção e das deformidades nos pés. Além das causas extrínsecas, nas quais estão relacionadas ao ambiente em que o idoso interage, em sua casa, em locais públicos e o transporte coletivo. ${ }^{3}$

Nesse contexto, os traumatismos ocorridos nos idosos, gerados por quedas, consistem em um problema de saúde pública. São frequentes devido a algumas debilidades comuns, existentes nessa fase da vida, tais 


\section{JOURNALOF \\ NURSING \\ ANo HEALTH}

ISSN 2236 - 1987

sul do Brasil?”. E possui como objetivo, identificar o perfil das idosas atendidas por queda em um serviço de emergência no sul do Brasil.

\section{MATERIAIS E MÉTODOS}

problemas de grande importância para as políticas públicas, no direcionamento de ações que objetivam promover melhoria para as condições de vida desse novo perfil brasileiro. Ademais, o país contém cinco regiões geográficas com variabilidade significativa em suas dimensões territoriais, sociais, econômicas e culturais. Desse modo, o envelhecimento também se manifesta segundo as diversidades regionais de cada uma delas. ${ }^{4}$

Assim, destaca-se a importância de inovação nos modelos assistenciais com o enfoque na prevenção, na detecção precoce e no tratamento das morbidades associadas à ocorrência de quedas. Para que haja benefícios para as pessoas idosas, de modo a melhorar sua qualidade de vida e reduzir os custos com essa ocorrência. ${ }^{3}$

Nesse contexto, torna-se importante para as equipes de saúde, em especial à enfermagem, conhecer o perfil da mulher idosa atendida por queda nos serviços de urgência e emergência. Dessa forma, salienta-se que a investigação realizada poderá trazer informações sobre as causas do acidente $e$ os fatores de risco predisponentes, o que facilitará o desenvolvimento de estratégias de prevenção de quedas e a promoção da saúde para os idosos.

Frente ao exposto, esse estudo lançou a seguinte questão norteadora: "Qual o perfil das idosas atendidas por queda em um serviço de emergência no
Trata-se de um estudo quantitativo, descritivo e transversal, desenvolvido em um Pronto-Socorro de um município no sul do Brasil. Essa instituição atende à população da Sistema Único de Saúde (SUS) e recebe diariamente uma média de 250 pacientes, conforme dados secundários obtidos junto às Fichas de Atendimento (FA). Atende a demanda espontânea e é referência em 23 municípios da Região Sul do Rio Grande do Sul, incluindo o Município sede.

A coleta de dados ocorreu de março a abril de 2010, sendo entrevistados 324 idosos que procuraram o Pronto-Socorro devido a causas externas. 0 cálculo do tamanho da amostra foi realizado no programa Epi Info 6.04. Para tanto, utilizou-se a população geral de Pelotas de acordo com estimativas do IBGE, sendo a porcentagem de idosos na população pelotense estimada em $14 \%$ e porcentagem de causas externas envolvendo idosos com uma estimativa de $7 \%$.

Os parâmetros utilizados foram: população de Pelotas: 339.934 habitantes; população com 60 anos ou mais em Pelotas: 14\%; prevalência de causas externas estimada em Pelotas: 7\%; nível de confiança: 95\%; erro aceitável na prevalência: 3 pontos percentuais; número de pessoas: 276 ; macrorregião, exclusivamente pelo 
acréscimo para perdas e recusas (+ 10\%): 304 .

Para este estudo, os sujeitos foram 153 mulheres idosas que responderam o instrumento de coleta de dados e que atenderam os seguintes critérios de inclusão: possuir idade igual ou superior a 60 anos e ter sofrido acidente por queda. As variáveis utilizadas foram: perfil demográfico, socioeconômico e situação de saúde.

Quanto ao perfil demográfico e socioeconômico, incluíram: idade (numérica continua); cor (categórica nominal); estado civil (categórica nominal); saber ler e escrever (categórica dicotômica); renda mensal (categórica nominal); aposentada (categórica dicotômica); trabalho (categórica dicotômica); e procedência (categórica nominal).

Em relação à situação de saúde, as variáveis foram: diabetes mellitus, hipertensão, reumatismo, problema de memória e nervosismo (categóricas dicotômicas); problemas de visão, audição e locomoção (categóricas dicotômicas); consumo de medicamentos (categórica dicotômica e numérica contínua): e história de quedas anteriores (categórica dicotômica).

Os dados coletados foram digitados no software Epi Info (versão 6.04), sob forma de dupla entrada, para análise da consistência interna. Realizou-se a análise descritiva, apresentando a distribuição do desfecho, de acordo com as variáveis independentes em um programa computacional.

Para a realização deste estudo, foram respeitados os preceitos da
Resolução 196/96 do Ministério da Saúde, em vigor no período, que trata de pesquisa envolvendo seres humanos. 50 projeto foi submetido ao Comitê de Ética em Pesquisa da Faculdade de Enfermagem da Universidade Federal de Pelotas, o qual foi aprovado sob o $n^{\circ} 59 / 2010$. Os sujeitos do estudo assinaram o Termo de Consentimento Livre e Esclarecido, em duas vias, com garantia do anonimato dos entrevistados e do direito de desistir a qualquer momento da pesquisa, sem prejuízo pessoal e/ou profissional.

\section{RESULTADOS}

Os resultados, a seguir, mostram - perfil socioeconômico e demográfico, assim como a situação de saúde das 153 idosas atendidas por quedas no Pronto-Socorro de Pelotas, no período de março a abril de 2010.

$\mathrm{Na}$ Tabela 1, apresenta-se dados relacionados ao perfil demográfico e socioeconômico da população estudada, como idade, cor autorreferida, estado civil, referência a saber ler e escrever, renda mensal, aponsentadoria e/ou atividades laborais e procedência.

$\mathrm{Na}$ Tabela 2, pode-se observar informações referentes à situação de saúde das idosas no que se refere a morbidades que elas afirmaram ser portadoras.

Já a Tabela 3 aponta dados relacionados aos problemas visuais, auditivos e locomotivos, além do consumo de medicamentos e da história prévia de quedas. 


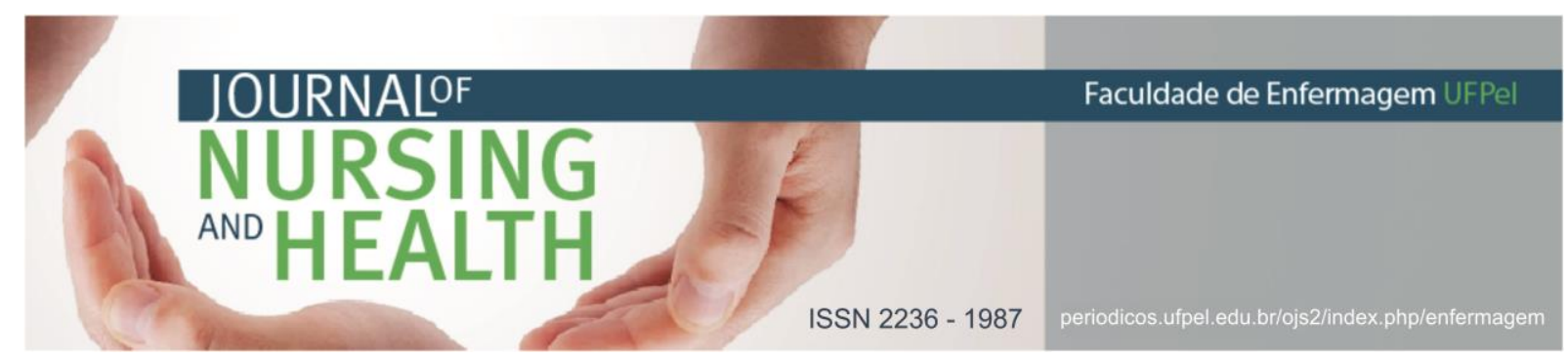

Tabela 1 - Perfil demográfico e socioeconômico das idosas atendidas por quedas no Pronto-Socorro Municipal de Pelotas. Pelotas/RS, Brasil, 2010

\begin{tabular}{|c|c|c|}
\hline & $(N=153)$ & $\%$ \\
\hline \multicolumn{3}{|l|}{ Idade } \\
\hline 60-64 anos & 37 & 24,1 \\
\hline 65-69 anos & 27 & 17,6 \\
\hline $70-80$ anos & 60 & 39,2 \\
\hline 81 e mais anos & 29 & 18,9 \\
\hline \multicolumn{3}{|l|}{ Cor } \\
\hline Branca & 124 & 81,0 \\
\hline Não Branca & 29 & 19,0 \\
\hline \multicolumn{3}{|l|}{ Estado civil } \\
\hline Casada ou com companheiro & 44 & 28,8 \\
\hline Solteira ou Separada & 38 & 24,8 \\
\hline Viúva & 71 & 46,4 \\
\hline \multicolumn{3}{|l|}{ Sabe ler e escrever } \\
\hline Não & 26 & 17,0 \\
\hline Sim & 127 & 83,0 \\
\hline \multicolumn{3}{|l|}{ Renda mensal* ${ }^{*}$} \\
\hline$\leq$ a um salário mínimo & 115 & 75,1 \\
\hline > que um salário mínimo & 38 & 24,84 \\
\hline \multicolumn{3}{|l|}{ Aposentada } \\
\hline Não & 35 & 22,9 \\
\hline Sim & 118 & 77,1 \\
\hline \multicolumn{3}{|l|}{ Trabalho } \\
\hline Não & 132 & 86,3 \\
\hline Sim & 21 & 13,7 \\
\hline \multicolumn{3}{|l|}{ Procedência } \\
\hline Zona rural & 11 & 7,2 \\
\hline Zona urbana & 142 & 92,8 \\
\hline
\end{tabular}

* Salário mínimo vigente em março/abril $2010 \mathrm{R} \$ 510,00$.

Tabela 2 - Situação de saúde das idosas que sofreram acidente por quedas e foram atendidas no Pronto-Socorro Municipal. Pelotas/RS, Brasil, 2010

\begin{tabular}{lrc}
\hline & $(\mathbf{N}=153)$ & $\%$ \\
\hline Diabetes Mellitus & 113 & 73,8 \\
Não & 40 & 26,1 \\
Sim & 59 & 38,5 \\
Hipertensão Arterial & 94 & 61,4 \\
Não & & 46,4 \\
Sim & 71 & 43,6 \\
Reumatismo & 82 & \\
Não & & 45,1 \\
Sim & 69 & 54,9 \\
Problema de memória & 84 & 39,2 \\
Não & & 60,8 \\
Sim & 60 & \\
Nervosismo & 93 & \\
Não & & \\
Sim & &
\end{tabular}


Tabela 3 - Problemas de visão, audição e locomoção, consumo de medicamentos e história de quedas anteriores. Pelotas/RS, Brasil, 2010

\begin{tabular}{lrc}
\hline & $(\mathrm{N}=153)$ & $\%$ \\
\hline $\begin{array}{l}\text { Problemas de Visão } \\
\text { Não }\end{array}$ & 29 & 18,9 \\
$\quad$ Sim & 124 & 81,1 \\
Problemas de Audição & 100 & 65,4 \\
$\quad$ Não & 53 & 34,6 \\
$\quad$ Sim & 57 & 37,2 \\
Problemas de locomoção & 96 & 62,7 \\
$\quad$ Não & & \\
Sim & 23 & 16,2 \\
Remédios diferentes tomados por dia & 43 & 30,3 \\
O a 01 & 72 & 50,7 \\
O2 a 03 04 ou mais & & \\
Queda da idosa nos últimos 12 meses & 61 & 39,9 \\
Não & 92 & 60,1 \\
Sim & & \\
\hline
\end{tabular}

\section{DISCUSSÃO}

Os achados desta pesquisa evidenciaram que as mulheres com idade entre 70 e 80 anos foram as que mais sofreram acidente por queda $(40 \%)$. Salienta-se que as mulheres apresentam maior longevidade quando comparadas aos homens. Esse fato as leva a períodos mais prolongados de vida, possibilitando o surgimento de alguma doença crônica não transmissível. Ademais, outros fatores podem ser incluídos, como a baixa renda, a perda do companheiro e a presença da solidão. De fato, as quedas acontecem mais em mulheres, e as causas para explicar a maior ocorrência no sexo feminino, ainda são pouco esclarecidas e controversas. ${ }^{6}$

A maioria das idosas se autodeclarou de cor branca, havendo no Brasil uma dificuldade dos indivíduos se declararem de outra cor, sendo a branca prevalente, mesmo em situações que ela não é evidente. Dado semelhante foi encontrado em uma pesquisa que objetivou conhecer a prevalência de quedas e verificar fatores associados a esse evento nos idosos institucionalizados no Município de Pelotas, no Rio Grande do Sul, sendo que $89,2 \%$ dos entrevistados também tinham a cor da pele branca. ${ }^{7}$

No presente estudo, foi constatado que quase metade das mulheres relatou ser viúva. Esse achado leva a refletir sobre a possibilidade de residirem sozinhas, apresentarem problemas visuais e de locomoção, o que acentuam na presença de quedas.

Em comparação com uma pesquisa que objetivou descrever 0 perfil epidemiológico de 65 idosos que participavam de grupos sociais no Estado do Ceará, 90,8\% eram mulheres, e a maioria das participantes afirmou serem viúvas, equivalendo a $44,6 \%$ da amostra. $^{8}$ Apesar do estudo ter sido realizado na região Sul do Brasil, a comparação com 


\section{JOURNALOF \\ NURSING \\ AND}

ISSN 2236 - 1987

o resultado de uma pesquisa da região Nordeste não apresentou diferença.

Mais de $80 \%$ das idosas deste estudo sabiam ler e escrever. Ao comparar com uma pesquisa realizada, $51,1 \%$ completaram o ensino fundamental, não sendo encontrada relação significativa com a queda e o nível de escolaridade. ${ }^{9}$ Já outro estudo desenvolvido em Ribeirão Preto, no Estado de São Paulo, encontrou um percentual de $14,6 \%$ do total de 240 participantes, cujo predomínio das quedas foram em idosas que declararam não serem letradas e 48,8\% frequentaram a escola entre 1 e 4 anos. ${ }^{10}$

Quanto à situação econômica, a maioria não trabalhava e $77 \%$ delas viviam somente com a aposentadoria, sendo que, em mais da metade, a remuneração equivalia, no máximo, a um salário mínimo. Outra pesquisa envolvendo acidentes por quedas em idosos ressaltou a probabilidade daqueles que possuem renda maior terem mais acesso a recursos médicos e mais conhecimento sobre a prevenção de alterações corporais, o que, direta ou indiretamente, pode levar à melhora de sua capacidade física. ${ }^{11}$

Com base no presente estudo, assim como em outras pesquisas relacionadas à mesma temática, se podem ter subsídios para as políticas públicas de atenção ao idoso. Principalmente para a mulher, no que tange à prevenção de quedas, considerando os seus fatores de risco.

Em relação às comorbidades, mais da metade das idosas, do presente estudo, eram hipertensas. Apesar da hipertensão arterial sistêmica não possuir relação para o aumento das quedas, trata-se de uma doença crônica comum entre os idosos e a sua prevalência aumenta progressivamente com a idade. Uma pesquisa realizada na cidade de Araraquara, Estado de São Paulo, evidenciou que a hipertensão arterial foi a doença de maior prevalência, apresentando-se em $66 \%$ da população investigada. ${ }^{12}$

Os achados neste estudo mostram que mais da metade das mulheres possuía problemas de reumatismo. Já uma pesquisa realizada, averiguou que os fatores de risco intrínsecos relacionados às doenças crônicas presentes nas vítimas de quedas, compreendeu a doença reumatológica/ortopédica em 100\% dos casos. Nesse sentindo, frisa-se que a elevação da incidência nas mulheres pode estar associada ao processo de declínio da massa óssea antes do homem, permitindo a geração de doenças reumatológicas/ortopédicas (osteopenia,

osteoartrose, osteoporose), estando essa ocorrência acentuada em idosas. ${ }^{13}$

Problema de nervosismo esteve presente em $60 \%$ das mulheres deste estudo. Esse fator também esteve presente em um estudo populacional com idosos sem déficit cognitivo em São Paulo, o qual objetivou avaliar a relação entre dor crônica e quedas relatadas pelos participantes. Dessa maneira, os autores afirmaram que houve associação de queda nos 12 meses posteriores a pesquisa com as doenças autorreferidas, dentre as quais problema nervoso. ${ }^{14}$

Em relação ao problema de memória, esse foi afirmado por $50 \%$ das idosas. Dado semelhante foi encontrado em uma pesquisa que 


\section{JOURNALOF \\ NURSING \\ AND HEALTH}

ISSN $2236-1987$

identificou a prevalência e os fatores associados a quedas e quedas recorrentes em uma amostra de 391 idosos no Município de Cuiabá/Mato Grosso, em que $74(50,7 \%)$ dos idosos que tiveram queda, também referiram ter dificuldade de memória. ${ }^{15}$

Nesse contexto, faz-se importante a promoção de segurança dentro e fora do domicílio, assim como manter os objetos e mobiliários em posições específicas para que a idosa não esbarre, e assim, diminuam os acidentes causados por fatores extrínsecos.

A acuidade visual é precária em aproximadamente $81 \%$ das idosas que foram atendidas no serviço de emergência onde foi realizado este estudo. Proporções menores foram encontradas em uma pesquisa de demanda, a qual avaliou o perfil de mulheres idosas com quedas, observando a presença de déficit visual em $52 \%$ da amostra. ${ }^{9}$ Nessa perspectiva, é importante orientar quanto às consultas oftalmológicas regulares, a fim de melhorar o campo visual e evitar os acidentes por queda.

No presente estudo, mais da metade das mulheres havia sofrido, pelo menos, uma queda no ano anterior. Esse achado se diferencia de uma pesquisa realizada com 78 mulheres, que dentre as participantes, $32,4 \%$ sofreram quedas no último ano, sendo menos da metade das que não sofreram $(67,6 \%){ }^{16}$

0 resultado, neste estudo, também demonstrou que cerca da metade das idosas que caíram, utilizava quatro ou mais fármacos. Salienta-se que, uma das causas intrínsecas relacionadas aos acidentes

por quedas em idosos, é o uso de polifármacos.

Situação essa semelhante a uma pesquisa realizada, em que o uso de medicações é comum na população idosa. Entretanto, naqueles que sofrem quedas, a presença de medicamento é maior, havendo predomínio dos anti-hipertensivos, dos hipoglicemiantes e dos psicotrópicos. Ainda foi encontrada, a utilização concomitante de duas ou mais classes de medicações, sendo caracterizada de polifarmácia. ${ }^{17}$

É essencial que o profissional médico, ao prescrever medicamentos, estabeleça uma avaliação criteriosa sobre a real necessidade do seu uso. Dada a estreita relação entre o uso de novos fármacos, ou mesmo, o ajuste de dosagem a cada seis meses, além do aumento no risco de quedas na população idosa.

A grande quantidade de medicações utilizadas pelos idosos concebe um fator preocupante, uma vez que os efeitos deletérios da interação medicamentosa são mais acentuados nessa faixa etária. Ressalva-se ainda, a necessidade frequente no acompanhamento do estado de saúde das pessoas idosas, no intuito de controlar de maneira eficaz, as doenças crônicas, e assim, reduzir o risco de agravos maiores. Também se destaca a importância da avaliação das medicações prescritas e 0 reconhecimento dos riscos da polifarmácia, já que esses fatores estão diretamente relacionados à ocorrência de quedas. ${ }^{17}$

Perante os dados encontrados neste estudo, destaca-se que identificar o perfil da população idosa vítima de quedas é importante para a 


\section{NURSING \\ AND}

ISSN 2236 - 1987

profissional, pois é necessário que políticas públicas visualizem essa população e que desenvolvam medidas de proteção para este público, pois como bem apresentado neste estudo, existem medidas extrínsecas que precisam ser contempladas para que as quedas sejam minimizadas. Assim, o poder público precisa ser responsabilizado pelo seu descuido com esta população.

\section{CONCLUSÃO}

A expectativa de vida vem aumentando consideravelmente, a partir da transição demográfica e epidemiológica, 0 que aumenta a probabilidade de acontecimentos causais como os acidentes por queda, principalmente nas mulheres idosas. Os resultados da pesquisa mostram que o perfil socioeconômico e demográfico das idosas atendidas no serviço de emergência por quedas era, predominantemente, composto por faixa etária entre 70 e 80 anos, de cor branca e viúvas. A grande maioria delas procedia da zona urbana, sabia ler e escrever, era aposentada e possuía renda de até um salário mínimo.

Em relação às doenças préexistentes, as idosas apresentaram hipertensão arterial, reumatismo, problemas de memória, de visão e nervosismo. A maioria tinha dificuldade para caminhar, fazia uso de polifármacos e relatou queda no ano anterior.

Por ser a queda um evento de causa multifatorial de alta complexidade terapêutica, acredita-se ser necessária a adoção de medidas individuais e coletivas para promover as condições de saúde dessa parcela da 


\section{JOURNALOF \\ NURSING \\ AND}

ISSN 2236 - 1987

2. Pinheiro Al, Almeida FM, Barbosa IV, Melo EM, Studart RMB, Carvalho ZMF. Principales causas asociadas al traumatismo craneoencefálico en ancianos. Enferm glob [Internet]. 2011 abr[acesso em 2014 jan 12];10(22). Disponível em: http://scielo.isciii.es/scielo.php?pid= S1695.

61412011000200005\&script=sci_arttex $\mathrm{t}$

3. Silva NVC, Borges MMMC. Perfil epidemiológico das mulheres idosas que apresentaram quedas na área de abrangência de uma superintendência regional de saúde. Revista Enfermagem Integrada. 2014;7(1):1220-30.

4. Santana da Silva LW, Squarcini CFR, Santos Júnior EB, Miranda da Silva N, Santos A, Pereira SFS, et al. Perfil de pessoas idosas com hipertensão arterial em um programa de atividade física com a família. Rev kairós. 2015 $\mathrm{jan} / \mathrm{mar}$;8(1):57-75.

5. Ministério da Saúde (BR). Conselho Nacional de Saúde. Resolução 196, de 10 de outubro de 1996: diretrizes e normas regulamentadoras de pesquisa envolvendo seres humanos. Brasília; 1996.

6. Ramos CV, Santos SSC, Barlem ELD, Pelzer MT. Quedas em idosos de dois serviços de pronto atendimento do Rio Grande do Sul. Rev eletrônica enferm [Internet]. 2011 out/dez[acesso em 2013 out 19];13(4):703-13. Disponível em:

https://www.fen.ufg.br/revista/v13/ n4/pdf/v13n4a15.pdf

7. Carvalho MP, Luckow ELT, Siqueira FV. Quedas e fatores associados em idosos institucionalizados no município de Pelotas (RS, Brasil). Ciênc saúde colet. 2011;16(6):2945-52. 


\section{NURSING \\ AND}

ISSN 2236 - 1987

quedas e quedas recorrentes em idosos: estudo de base populacional. Rev bras geriatr gerontol. 2014;17(1):49-60.

16. Vitor PRR, Oliveira ACK, Kohler R, Winter GR, Rodacki C, Krause PM. Prevalência de quedas em mulheres idosas. Acta ortop bras. 2015;23(3):158-61.

17. Costa AGS, Souza RC, Vitor AF, Araujo TL. Acidentes por quedas em um grupo específico de idosos. Rev eletrônica enferm [Internet]. 2011 jul/set[acesso em 2014 jan 29];13(3):395-404. Disponível em: https://www.fen.ufg.br/fen_revista/ v13/n3/pdf/v13n3a04.pdf

18. Costa AMR, Xavier EMO, Filgueiras MC. Perfil epidemiológico de idosos com fraturas atendidos em hospital de emergência. Rev bras ciênc saúde. 2012 out/dez; 10(34):41-6.

19. Cavalcante ALP, Aguiar JB, Gurgel LA. Fatores associados a quedas em idosos residentes em um bairro de Fortaleza, Ceará. Rev bras geriatr gerontol. 2012;15:(1)137-46.

20. Gautério DP, Vidal DAS, Barlem JGT, Santos SSC. Ações educativas do enfermeiro para a pessoa idosa: Estratégia Saúde da Família. Rev enferm UERJ. 2013;21(6):824-8.

Data da submissão: 2016-01-29

Aceito: 2017-06-24

Publicação: 2017-04-12

14. Dellaroza MSG, Pimenta CAM, Lebrão $\mathrm{ML}$, Duarte $\mathrm{YAO}$, Braga $\mathrm{P}$. Associação entre dor crônica e autorrelato de quedas: estudo populacional - SABE. Cad saúde pública. 2014;30(3):522-32.

15. Soares WJS, Moraes SA, Ferriolli E, Perracini MR. Fatores associados a 